\title{
THE GEOMETRY OF UNIFORM, QUASICIRCLE, AND CIRCLE DOMAINS
}

\author{
DAVID A. HERRON
}

Abstract. We introduce a class of collared domains. We solve the Kreisnormierungsproblem for collared quasicircle domains in $\mathbf{R}^{2}$. Then we give geometric conditions which ensure that a quasicircle domain be collared and in fact be uniform. Finally, we state our results in terms of hyperbolic geometry.

\section{Introduction}

Throughout this paper $D$ denotes a domain in euclidean $n$-space $\mathbf{R}^{n}$. We are primarily interested in the plane $\mathbf{R}^{2}$, which we identify with $\mathbf{C}$, so except where explicitly noted otherwise we always have $n=2$.

We call $D$ uniform if there is a constant $c$ such that each pair of points $z_{1}, z_{2}$ in $D$ can be joined by an arc $\alpha$ in $D$ satisfying

and

$$
l(\alpha) \leqq c\left|z_{1}-z_{2}\right|
$$

$$
\min _{j=1,2} l\left(a_{j}\right) \leqq c d(z, \partial D) \text { for all } z \in \alpha .
$$

Here $l(\alpha)$ is the euclidean length of $\alpha, d(\cdot, \cdot)$ is euclidean distance, and $\alpha_{1}, \alpha_{2}$ are the components of $\alpha-\{z\}$. Condition (1) says that $l(\alpha)$ is comparable to $\left|z_{1}-z_{2}\right|$; condition (2) says that away from $z_{1}, z_{2} \alpha$ stays away from $\partial D$. Martio and Sarvas first introduced uniform domains [MS] and since then they have appeared quite naturally throughout function theory. Gehring has a recent survey article [G3] which lists many of the properties of uniform domains and has an extensive bibliography.

There are numerous alternative descriptions of uniform domains, however, with all of these it is often a non-trivial task to verify that a domain is uniform. In Theorem

KEYWORDS: uniform domains, quasicircle domains, circle domains, rings, quasiconformal mappings, hyperbolic metric

1980 Mathematics Subject Classification (1985 Revision): Primary 30C60, Secondary 30C20 
1.2 we give sufficient conditions for a domain to be uniform. These are geometric conditions in terms of the domain's boundary components and often can easily be verified. As a byproduct of the proof of Theorem 1.2 we solve a special case of the Kreisnormierungsproblem; see Theorem 2.6.

As the following result indicates, the complementary regions determined by a uniform domain in the plane must satisfy certain geometric conditions. It is not hard to see that the boundary components of a uniform domain must be linearly locally connected and hence are quasicircles [MS, Corollary 2.33], [G1, Theorem 4.1]. We call $D$ a $K$-quasicircle domain if each of its boundary components is either a point or a $K$-quasicircle.

1.1. Theorem. Suppose $D$ is uniform. Then $D$ is a K-quasicircle domain, $K=K(c)$, and there exists $a$ constant $a=a(c)$ such that

$$
\min _{j=1,2} \operatorname{dia}\left(B_{j}\right) \leqq a d\left(B_{1}, B_{2}\right)
$$

for any two components $B_{1}, B_{2}$ of $\overline{\mathbf{C}}-D$.

Condition (3) says that any two complementary regions determined by a uniform domain cannot be too close together unless one of them is small [G2, Theorem 2 and Lemma 3]; this condition can be derived directly from conditions (1) and (2).

Thus a simply connected proper subdomain of the plane is uniform if and only if it is a quasidisk, and in general a uniform domain is a quasicircle domain. In fact, a quasicircle domain is quasiconformally equivalent to a uniform domain if and only if it itself is uniform [GM, Corollary 3.17]. So whether or not a plane domain is uniform depends on two criteria: First, its boundary components must have the right shape; they must be quasicircles or points. Second, its boundary components must be in the right position with respect to each other; the internal moduli of the domain must satisfy appropriate conditions.

It is easily seen that condition (3) is not strong enough to guarantee that $D$ be uniform, e.g., consider the quasicircle domain $\mathbf{C}-\mathbf{Z}$. However, we do have the following result.

1.2. The orem. Let $B_{0}$ be the component of $\overline{\mathbf{C}}-D$ which contains $\infty$. Suppose $D$ is a $K$-quasicircle domain and there exist constants $a, b$ such that

$$
\operatorname{dia}\left(B_{1}\right) \leqq \operatorname{ad}\left(B_{1}, B_{0}\right)
$$

and

$$
\min _{j=1,2} d\left(B_{j}, B_{0}\right) \leqq b d\left(B_{1}, B_{2}\right)
$$

for any two components $B_{1}, B_{2}$ of $\overline{\mathbf{C}}-D-B_{0}$. Then $D$ is uniform with $c=c(a, b, K)$.

Condition (4) is a weak-version of (3) and thus is necessary for $D$ to be uniform. Condition (5) says that $B_{0} \neq\{\infty\}$ and that any two bounded complementary regions determined by $D$ cannot be too close together unless they are close to $B_{0}$ (and hence 
by (4) small). It is easy to see that there are uniform $D$ which do not satisfy (5); e.g. $D=\{z \in \mathbf{C}: \operatorname{Re}(z)>0\}-\left\{2^{n}, 2^{n}+1: n=1,2, \ldots\right\}$.

We prove Theorem 1.2 in Section 3. It turns out that we need only consider the special case of circle domains which have points and circles for boundary components. The general case follows from a result on quasiconformal mappings, Theorem 2.6, together with the quasi-invariance of the hypotheses.

In Section 2 we state and prove Theorem 2.6. In particular, this theorem gives sufficient conditions for the existence of a conformal mapping of a quasicircle domain onto a circle domain.

In Section 4 we conclude by stating our results in terms of hyperbolic geometry.

The author wishes to thank Professor F. W. Gehring for suggesting this problem and for many helpful conversations. The author also thanks the referee for numerous helpful comments.

\section{Extension of quasiconformal mappings}

Our ultimate result here, Theorem 2.6, is that certain quasicircle domains can be mapped conformally onto circle domains via quasiconformal self-homeomorphisms of $\overline{\mathbf{C}}$.

2.1. Notation. We use the following standard notation. Let $\Gamma$ be a nonempty family of curves. We call $\varrho$ admissible for $\Gamma$, denoted $\varrho \in \operatorname{adm}(\Gamma)$, if $\varrho$ is non-negative and Borel measurable and if $\int_{\gamma} \varrho(z)|d z| \geqq 1$ for each locally rectifiable $\gamma \in \Gamma$. The modulus of $\Gamma$ is $M(\Gamma)=\inf _{\varrho} \iint \varrho(z)^{2} d x d y$, where the infimum is taken over all $\varrho \in \operatorname{adm}(\Gamma)$.

A ring is a doubly connected domain; thus every ring has exactly two boundary components. The modulus of a ring $R$ is defined by

$$
\bmod (R)=2 \pi M\left(\Gamma_{1}\right)=2 \pi / M\left(\Gamma_{2}\right),
$$

where $\Gamma_{1}, \Gamma_{2}$ are the families of curves in $R$ which respectively separate, join the boundaries of $R$. The extremal ring of Grötzsch is $\{z \in \mathbf{C}:|z|<1\}-[0, r]$ and its modulus is denoted by $\mu(r), 0<r<1$. See [LV].

Two lemmas, each of independent interest, are required for the proof of Theorem 2.6. For each of these lemmas we use the following notation: $R$ is a ring with boundary components $C_{0}$ and $C, C$ is a Jordan curve, and $D$ and $D_{0}$ are respectively the components of $\overline{\mathbf{C}}-C$ and $\overline{\mathbf{C}}-C_{0}$ containing $R$.

2.2. Lemma. Let $\alpha$ and $\beta$ be disjoint arcs in $C$ and let $\Gamma_{R}, \Gamma_{D}$ be the families of curves in $R, D$ respectively which join $\alpha$ and $\beta$. Then

$$
M\left(\Gamma_{R}\right) \leqq M\left(\Gamma_{D}\right) \leqq \varphi(\bmod R) M\left(\Gamma_{R}\right),
$$

where $\varphi(t)=\left[1+\exp \left(\pi^{2} / 2 t\right)\right] / 2$. 
Proof. As $\Gamma_{R} \subset \Gamma_{D}, \quad M\left(\Gamma_{R}\right) \leqq M\left(\Gamma_{D}\right)$ and we need only prove the second inequality.

Map $D$ conformally onto a rectangle $D_{1}$ so that $\alpha$ and $\beta$ are sent onto the vertical sides $[-a-i,-a+i]$ and $[a-i, a+i]$. Let $R_{1}, C_{1}, \Gamma_{R_{1}}, \Gamma_{D_{1}}$ be the images of $R, C_{0}, \Gamma_{R}, \Gamma_{D}$ respectively. Note that $M\left(\Gamma_{D}\right)=M\left(\Gamma_{D_{1}}\right)=1 / a$.

Let $\left[i y_{1}, i y_{2}\right] \subset[-i, i]$ be the projection of $C_{1}$ onto the imaginary axis. For each $y \in(-1,1)-\left[y_{1} y_{2}\right], \gamma_{y}=\{x+i y:-a<x<a\} \in \Gamma_{R_{1}}$, so for each $\varrho \in \operatorname{adm}\left(\Gamma_{R_{1}}\right)$

whence

$$
1 \leqq\left(\int_{\gamma_{y}} \varrho(z)|d z|\right)^{2}=\left(\int_{-a}^{a} \varrho(x+i y) d x\right)^{2} \leqq 2 a \int_{-a}^{a} \varrho(x+i y)^{2} d x,
$$

$$
\iint \varrho(z)^{2} d x d y \geqq\left(\int_{-1}^{y_{1}}+\int_{y_{2}}^{1}\right) d y /(2 a)=\left[2-\left(y_{2}-y_{1}\right)\right] /(2 a) .
$$

Taking the infimum over all $\varrho \in \operatorname{adm}\left(\Gamma_{R_{1}}\right)$ yields $M\left(\Gamma_{R_{1}}\right) \geqq\left[1-\left(y_{2}-y_{1}\right) / 2\right] M\left(\Gamma_{D_{1}}\right)$, whence

$$
M\left(\Gamma_{D}\right) \leqq c M\left(\Gamma_{R}\right), c=1 /\left[1-\left(y_{2}-y_{1}\right) / 2\right] .
$$

Let $R_{2}$ be the Steiner symmetrization of $R_{1}$ with respect to the imaginary axis [H], let $R_{3}$ be the ring with boundary components $C_{3}=\left[i y_{1}, i y_{2}\right]$ and $\{x \pm i: x \in \overline{\mathbf{R}}\}$, and let $R_{4}=\psi \circ \varphi\left(R_{3}\right)$ where

$$
\varphi(z)=\tanh \left(\frac{\pi z}{4}\right), \quad \psi(w)=\frac{w-i v_{1}}{1+i v_{1} w}, \quad i v_{j}=\varphi\left(i y_{j}\right)=i \tan \left(\frac{\pi y_{j}}{4}\right) \quad(j=1,2) .
$$

Then

$$
m=\bmod (R)=\bmod \left(R_{1}\right) \leqq \bmod \left(R_{2}\right) \leqq \bmod \left(R_{3}\right)=\bmod \left(R_{4}\right)=\mu(t),
$$

where $i t=\psi\left(i v_{2}\right)$. Next,

$$
\mu^{-1}(m) \geqq t=\frac{\tan s_{2}-\tan s_{1}}{1-\tan s_{1} \tan s_{2}} \geqq \sin \left(s_{2}-s_{1}\right),
$$

where $s_{j}=(\pi / 4) y_{j}(j=1,2)$. Thus

$$
y=\left(y_{2}-y_{1}\right) / 2 \leqq \sin (\pi y / 2) \leqq \mu^{-1}(m),
$$

so [LV, (2.9), p. 61]

Finally,

$$
\log \frac{1+y}{1-y} \leqq \mu\left(\frac{1-y}{1+y}\right)=\frac{\pi^{2}}{2 \mu(y)} \leqq \frac{\pi^{2}}{2 m} .
$$

$$
c=1 /(1-y)=\left(1+\frac{1+y}{1-y}\right) / 2 \leqq\left[1+\exp \left(\pi^{2} / 2 m\right)\right] / 2 .
$$

2.3. Lemma. Suppose that $C$ is a $K$-quasicircle. Let $f$ be a $K_{0}$-quasiconformal mapping of $R$, and suppose that the boundary component $C^{\prime}$ of $R^{\prime}=f(R)$ which corresponds to $C$ under $f$ is a $K^{\prime}$-quasicircle. Then $f$ extends to a quasiconformal mapping of $D_{0}$ with maximal dilatation bounded above by a constant which depends only on $K_{0}, K, K^{\prime}$ and $M(R)=2 \pi / \bmod (R)$. 
Proof. Let $g, g^{\prime}: \overline{\mathbf{C}} \rightarrow \overline{\mathbf{C}}$ be $K, K^{\prime}$-quasiconformal mappings of $C, C^{\prime}$ onto the real axis with $g(R), g^{\prime}\left(R^{\prime}\right)$ lying in the upper half plane. To get the desired extension of $f$ it suffices to extend $g^{\prime} \circ f \circ g^{-1}$ to the lower half plane. Thus we may assume that $R$ and $R^{\prime}$ are rings in the upper half plane $D=D^{\prime}$ each having the real axis as a boundary component and that $f: R \rightarrow R^{\prime}$ is $K_{0}$-quasiconformal with $f: \partial D \rightarrow \partial D^{\prime}$. To extend $f$ to the lower half plane we need only show [LV, Theorem II.6.1] that there exists a constant $a>0$, which depends only on $K_{0}$ and $M(R)$, such that for all $x \in \mathbf{R}$ and for all $t>0$ we have

$$
1 / a \leqq M\left(\Gamma_{D^{\prime}}\right) \leqq a,
$$

where $\Gamma_{D^{\prime}}$ is the family of curves in $D^{\prime}$ joining the intervals $[f(x-t), f(x)]$; $[f(x+t), f(\infty)]$.

So fix $x \in \mathbf{R}$ and $t>0$. Let $\alpha$ and $\beta$ be the intervals $[f(x-t), f(x)]$ and [ $f(x+t), f(\infty)]$. Then let $\Gamma_{R^{\prime}}$ and $\Gamma_{D^{\prime}}$ be the families of curves in $R^{\prime}$ and $D^{\prime}$ respectively which join $\alpha, \beta$ and let $\Gamma_{R}$ and $\Gamma_{D}$ be the corresponding families of curves in $R$ and $D$. By Lemma $2.2 \quad 1=M\left(\Gamma_{D}\right) \leqq \varphi(\bmod R) M\left(\Gamma_{R}\right)$, and thus, letting $a=$ $K_{0} \varphi(2 \pi / M(R))$, we get

$$
1 / a \leqq\left(1 / K_{0}\right) M\left(\Gamma_{R}\right) \leqq M\left(\Gamma_{R^{\prime}}\right) \leqq M\left(\Gamma_{D^{\prime}}\right),
$$

which gives the first inequality in (6). The second inequality follows by symmetry.

2.4. Corollary. If the boundary components of a ring are quasicircles, then the ring is a quasiannulus, i.e., the image of an annulus under a quasiconformal selfhomeomorphism of $\overline{\mathbf{C}}$.

Proof. Let $R$ be a ring with boundary components the $K$-quasicircles $C_{0}$ and $C$. Let $D$ be the component of $\overline{\mathbf{C}}-C$ containing $R$. Choose a conformal mapping $f$ of $R$ onto an annulus $A$. Using Lemma 2.3 we extend $f$ to a $K^{\prime}$-quasiconformal mapping $g$ of $D$ where $K^{\prime}=K^{\prime}(K, M(R))$. We extend $g$ by reflection to a $K^{2} K^{\prime}$ quasiconformal mapping $h$ of $\overline{\mathbf{C}}$. Then $R=h^{-1}(A)$ is a $K^{2} K^{\prime}$-quasiannulus.

2.5. Collars. A component $B$ of $\mathbf{C}-D$ is said to have a collar of modulus $m$ in $D$ if there exists a ring in $D$ with modulus at least $m$ and having $\partial B$ as a boundary component. Let $B_{0}$ be a fixed component of $\overline{\mathbf{C}}-D$. We say that $D$ is $m$-collared relative to $B_{0}$ if each component $B$ of $\mathbf{C}-D-B_{0}$ has a collar of modulus $m$ in $D$ such that the collars are pairwise disjoint.

2.6. Theorem. Let $D$ be a $K$-quasicircle domain. Let $B_{0}$ be a component of $\overline{\mathbf{C}}-D$ with $\partial B_{0}$ a quasicircle. Suppose that $D$ is m-collared relative to $B_{0}$ with $m>0$. Then there exists a $K^{\prime}$-quasiconformal self-homeomorphism of $\overline{\mathbf{C}}$ which maps $D$ conformally onto a circle domain, where $K^{\prime}=K^{\prime}(K, m)$.

Proof. We assume that $B_{0}$ is the lower half-plane $\overline{\mathbf{C}}-U$ and that $i \in D$. Let $B_{1}, B_{2}, \ldots$ denote the other components of $\overline{\mathbf{C}}-D$. For $n=1,2, \ldots$ let $D_{n}=$ 
$U-\bigcup_{j=1}^{n} B_{j}$ and choose a conformal mapping $f_{n}: D_{n} \rightarrow D_{n}^{\prime}=U-\bigcup_{j=1}^{n} B_{j}^{(n)}$ of $D_{n}$ onto a circle domain $D_{n}^{\prime}$. Here each $B_{j}^{(n)}$ is a closed disk or a point and $\partial U, \partial B_{j}$, $i$ correspond to $\partial U, \partial B_{j}^{(n)}, i$. See Goluzin's text [G, Theorem 2, p. 237] or the original work of Koebe for the existence of the mappings $f_{n}$.

Next, for $j=1,2, \ldots, n$ let $R_{j}^{(n)}=f_{n}\left(R_{j}\right)$, where $R_{j}$ is the collar associated with $B_{j}$. By Lemma 2.3 there exists a $K^{\prime}$-quasiconformal extension $F_{n}^{(j)}: R_{j} \cup$ $B_{j} \rightarrow R_{j}^{(n)} \cup B_{j}^{(n)}$ of $f_{n} \mid R_{j}, K^{\prime}=K^{\prime}(K, m)$. Then

$$
F_{n}= \begin{cases}F_{n}^{(j)} & \text { in } R_{j} \cup B_{j} \\ f_{n} & \text { elsewhere }\end{cases}
$$

defines a $K^{\prime}$-quasiconformal self-homeomorphism of $U$ with $F_{n} \mid D_{n}=f_{n}$.

Thus we have a sequence of $K^{\prime}$-quasiconformal mappings $F_{n}: U \rightarrow U$ with $F_{n} \mid D$ conformal, $F_{n}(i)=i, F_{n}: \partial U \rightarrow \partial U$. By normality [LV, Theorem II.5.1] there exists a subsequence, which we continue to call $\left\{F_{n}\right\}$, which converges uniformly on compact subsets of $U$ to some function $F$. Since $F(i)=i, F$ must be a $K^{\prime}$-quasiconformal self-homeomorphism of $U$ [LV, Theorem II.5.5].

We extend $F$ to $\overline{\mathbf{C}}$ by reflection. It remains to show that $F(D)$ is a circle domain. Write

$$
F(D)=D^{\prime}=U-\cup_{j=1}^{\infty} B_{j}^{\prime}, B_{j}^{\prime}=F\left(B_{j}\right) .
$$

Fix a $B_{j}^{\prime}$ and assume it is not a point. Fix $w_{1}, w_{2}, w_{3}, w_{4} \in \partial B_{j}^{\prime}$. For $k=1,2,3,4$ choose $z_{k} \in \partial B_{j}$ so that $w_{k}=F\left(z_{k}\right)$ and let $w_{k}^{(n)}=F_{n}\left(z_{k}\right)$. Since $\partial B_{j}^{(n)}$ is a circle, it follows that the cross ratio

$$
\left[w_{1}, w_{2}, w_{3}, w_{4}\right]=\lim _{n \rightarrow \infty}\left[w_{1}^{(n)}, w_{2}^{(n)}, w_{3}^{(n)}, w_{4}^{(n)}\right]
$$

must be real. Thus $\partial B_{j}^{\prime}$ is a circle, and $D^{\prime}$ is a circle domain.

\section{Proof of Theorem 1.2}

The simplest geometric situation to consider is when $D$ is a circle domain. We prove Theorem 1.2 for such domains and then appeal to Theorem 2.6 for the general case. The success of this strategy depends on showing that Theorem 2.6 applies and that the hypotheses of Theorem 1.2 are quasi-invariant with respect to quasiconformal self-homeomorphisms of $\mathbf{C}$.

The following result is useful [GO, Lemma 3].

3.1. Lemma. There exists a constant $\beta=\beta(n, K)$ such that for any $K$-quasiconformal $f: \mathbf{R}^{n} \rightarrow \mathbf{R}^{n}$

$$
\frac{\left|f\left(x_{1}\right)-f\left(x_{0}\right)\right|}{\left|f\left(x_{1}\right)-f\left(x_{2}\right)\right|}+1 \equiv \beta\left(\frac{\left|x_{1}-x_{0}\right|}{\left|x_{1}-x_{2}\right|}+1\right)^{1 / \alpha}, \quad \alpha=K^{1 /(1-n)}
$$

for all $x_{0}, x_{1}, x_{2} \in \mathbf{R}^{n}$. 
The next two results show that the hypotheses of Theorem 1.2 are quasi-invariant with respect to quasiconformal self-homeomorphisms of $\mathbf{R}^{n}$.

3.2. Lemma. Let $B_{0}, B_{1} \subset \mathbb{R}^{n}$ be closed. Suppose condition (4) holds. Then there exists a constant $b=b(a, n, K)$ such that

$$
\operatorname{dia}\left(f B_{1}\right) \leqq b d\left(f B_{1}, f B_{0}\right)
$$

for any $K$-quasiconformal $f: \mathbf{R}^{n} \rightarrow \mathbf{R}^{n}$.

Proof. Choose points $y_{1}=f\left(x_{1}\right), y_{2}=f\left(x_{2}\right) \in f\left(B_{1}\right)$ and $y_{0}=f\left(x_{0}\right) \in f\left(B_{0}\right)$ so that $\left|y_{1}-y_{0}\right|=d\left(f B_{1}, f B_{0}\right), 2\left|y_{1}-y_{2}\right| \geqq d\left(f B_{1}\right)$. Then by Lemma 3.1

$$
\frac{\operatorname{dia}\left(f B_{1}\right)}{d\left(f B_{1}, f B_{2}\right)} \leqq 2 \frac{\left|y_{1}-y_{2}\right|}{\left|y_{1}-y_{0}\right|} \leqq 2\left[\beta\left(\frac{\left|x_{1}-x_{2}\right|}{\left|x_{1}-x_{0}\right|}+1\right)^{1 / \alpha}-1\right] \leqq 2\left[\beta(a+1)^{1 / \alpha}-1\right] .
$$

3.3. Lemma. Let $B_{0}, B_{1}, B_{2} \subset \mathbf{R}^{n}$ be closed. Suppose conditions (4) and (5) hold. Then there exists a constant $c=c(a, b, n, K)$ such that

$$
\min _{j=1,2} d\left(f B_{j}, f B_{0}\right) \leqq c d\left(f B_{1}, f B_{2}\right)
$$

for any $K$-quasiconformal $f: \mathbf{R}^{n} \rightarrow \mathbf{R}^{n}$.

Proof. For $j=1,2$ choose points $y_{j}=f\left(x_{j}\right) \in f\left(B_{j}\right)$ so that $d\left(f B_{1}, f B_{2}\right)=\left|y_{1}-y_{2}\right|$. Assume that $d\left(B_{1}, B_{0}\right) \leqq d\left(B_{2}, B_{0}\right)$. Choose $x_{0} \in B_{0}$ with $d\left(B_{1}, B_{0}\right)=d\left(B_{1}, x_{0}\right)$. Then using (4) and (5) we obtain

$$
\left|x_{1}-x_{0}\right| \leqq \operatorname{dia}\left(B_{1}\right)+d\left(B_{1}, B_{0}\right) \leqq(a+1) d\left(B_{1}, B_{0}\right) \leqq b(a+1) d\left(B_{1}, B_{2}\right) .
$$

Thus by Lemma 3.1

$$
\frac{\min _{j=1,2} d\left(f B_{j}, f B_{0}\right)}{d\left(f B_{1}, f B_{2}\right)} \leqq \frac{\left|y_{1}-f\left(x_{0}\right)\right|}{\left|y_{1}-y_{2}\right|} \leqq \beta\left(\frac{\left|x_{1}-x_{0}\right|}{\left|x_{1}-x_{2}\right|}+1\right)^{1 / \alpha}-1 \leqq \beta(b(a+1)+1)^{1 / x}-1 .
$$

The corollary of the next lemma ensures that Theorem 2.6 can be applied; the lemma is also needed in the proof of Theorem 1.2 for a circle domain.

3.4. Lemma. Let $B_{0}, B_{1}, B_{2} \subset \mathbf{R}^{n}$ be closed. Suppose conditions (4) and (5) hold. Then

$$
\max _{j=1,2} d\left(B_{j}, B_{0}\right) \leqq(1+a b+b) d\left(B_{1}, B_{2}\right) .
$$

Proof. Suppose that $d\left(B_{1}, B_{0}\right) \leqq d\left(B_{2}, B_{0}\right)$. Then by (4), (5) and the triangle inequality

$$
\begin{gathered}
d\left(B_{2}, B_{0}\right) \leqq d\left(B_{1}, B_{0}\right)+\operatorname{dia}\left(B_{1}\right)+d\left(B_{1}, B_{2}\right) \\
\leqq(1+a) d\left(B_{1}, B_{0}\right)+d\left(B_{1}, B_{2}\right) \leqq((1+a) b+1) d\left(B_{1}, B_{2}\right) .
\end{gathered}
$$

3.5. Corollary. Assume the hypotheses of Theorem 1.2. Then $D$ is m-collared relative to $B_{0}$, where $m=m(a, b)>0$. 
Proof. To each component $B$ of $\overline{\mathbf{C}}-D-B_{0}$ we associate the ring

$$
R=\left\{z \in \mathbf{C}-B: d(z, B)<\varepsilon d\left(B, B_{0}\right)\right\},
$$

where $2 \varepsilon=1 /(1+a b+b)$. By Lemma 3.4 these rings are contained in $D$ and are pairwise disjoint. We claim that $\bmod (R) \geqq 2 /(1+a / \varepsilon)^{2}$.

Let $r=\operatorname{dia}(B), s=\varepsilon d\left(B, B_{0}\right)$. Note that $r / s \leqq a / \varepsilon$. Fix $z_{0} \in B$. Define

$$
\varrho(z)= \begin{cases}1 / s & \text { in } B\left(z_{0}, r+s\right) \\ 0 & \text { elsewhere. }\end{cases}
$$

Then $\varrho \in \operatorname{adm}(\Gamma)$ where $\Gamma$ is the family of curves in $R$ joining its boundary components. Thus $M(\Gamma) \leqq \iint \varrho^{2}=\pi(1+r / s)^{2}$, whence $\bmod (R)=2 \pi / M(\Gamma) \geqq 2 /(1+a / \varepsilon)^{2}$.

We require the following two results about annuli whose proofs are left for the reader. We remark that the necessary curves can be obtained by using straight line segments together with arcs of circles.

3.6. Lemma. An annulus $A$ is uniform with $c=O(1), O\left(\left(1-e^{-m}\right)^{-1}\right)$ as $m=$ $\bmod (A) \rightarrow \infty, 0$ respectively.

3.7. Lemma. Let $A$ be an annulus with inner, outer boundary circles $C_{1}, C_{2}$ respectively. Then points $z_{1} \in A, z_{2} \in C_{2}$ can be joined by an arc $\sigma$ in $A \cup C_{2}$ such that for every $z \in \sigma$

$$
l\left(\sigma\left(z_{1}, z\right)\right) \leqq \min \left\{c_{0}\left|z_{1}-z\right|, b_{0} d\left(z, C_{1}\right)\right\},
$$

where $c_{0}=2(1+\pi), b_{0}=O(1), O\left(e^{m}\right)$ as $m=\bmod (A) \rightarrow \infty, 0$ respectively.

Here, and below in 3.8 , we use the notation $\gamma(z, w)$ to denote the subarc between $z$ and $w$ of an $\operatorname{arc} \gamma$.

3.8. Proof of Theorem 1.2. By appealing to Theorem 2.6 and the quasi-invariance of our hypothesis with respect to quasiconformal self-homeomorphisms of $\mathbf{C}$, we may assume that $B_{0}=\overline{\mathbf{C}}-U$ and that $D=U-\bigcup_{j=1}^{\infty} B_{j}$. Here each $B_{j}$ is a closed euclidean disk or a point in $U$ and either $U$ is the open upper half-plane if $\infty \in \partial B_{0}$ or $U$ is the open unit disk if $\infty$ is an interior point of $B_{0}$.

Set $r_{j}=\operatorname{dia}\left(B_{j}\right), d_{j}=d\left(B_{j}, B_{0}\right)$ and let $A_{j}=\left\{z \in \mathbf{C}-B_{j}: d\left(z, B_{j}\right)<\varepsilon d_{j}\right\}$ where $4 \varepsilon=1 /(1+a b+b)$. Then as in the proof of Corollary 3.5 we find that we have disjoint annuli $A_{j}$ in $D$ with $\partial A_{j}=\partial B_{j} \cup C_{j}$ and $\bmod \left(A_{j}\right) \geqq m=m(a, b)>0$. Also, we see that if $z \in \bar{A}_{j}$, then $d(z, \partial D)=d\left(z, B_{j}\right)$. Moreover, if $z \in D$ and $d(z, \partial D)=d\left(z, B_{0}\right)$, then $z \notin \bar{A}_{j}$.

Fix $z_{1}, z_{2} \in D$. We must exhibit an arc $\alpha$ in $D$ which joins $z_{1}, z_{2}$ and satisfies (1), (2). By Lemma 3.6 we may assume that $z_{1}, z_{2}$ do not both lie in some annulus $A_{j}$. By relabeling, if necessary, we may assume that $d\left(z_{k}, \partial D-B_{0}\right)=d\left(z_{k}, B_{k}\right)$ for $k=1,2$. Let $\beta$ be the subarc of the circle through $z_{1}, z_{2}$ which is orthogonal to $\partial U$.

If $z_{k} \in A_{k}$, choose $w_{k} \in \beta \cap C_{k}$ and let $\sigma_{k}$ be the curve given by Lemma 3.7 which joins $z_{k}$ and $w_{k}$ in $A_{k} \cup C_{k}$; otherwise set $w_{k}=z_{k}$ and $\sigma_{k}=\left\{z_{k}\right\}$. Next, let $J$ denote 
the set of all $j$ for which $\beta$ meets $C_{j}$ twice, and for each $j \in J$ : let $\zeta_{1 j}, \zeta_{2 j}$ be the points of $\beta \cap C_{j}$ with $\zeta_{k j}$ the point first encountered as $\beta$ is traversed from $z_{k}$, let $\beta_{j}=\beta\left(\zeta_{1 j}, \zeta_{2 j}\right)$, and let $\gamma_{j}$ be the component of $C_{j}-\left\{\zeta_{1 j}, \zeta_{2 j}\right\}$ with minimal euclidean length. Finally, let

$$
\alpha=\sigma_{1} \cup\left[\left(\beta\left(w_{1}, w_{2}\right)-\bigcup_{J} \beta_{j}\right) \cup \bigcup_{J} \gamma_{j}\right] \cup \sigma_{2} .
$$

We show that $\alpha$ satisfies (1) and (2).

By Lemma 3.7 for $k=1,2$

$$
l\left(\sigma_{k}\right) \leqq c_{0}\left|z_{k}-w_{k}\right| \leqq c_{0} l\left(\beta\left(z_{k}, w_{k}\right)\right),
$$

and by geometry for all $j \in J$

$$
l\left(\gamma_{j}\right) \leqq \pi / 2\left|\zeta_{1 j}-\zeta_{2 j}\right| \leqq \pi / 2 l\left(\beta_{j}\right) .
$$

Since $c_{0} \geqq \pi / 2$, we conclude that

$$
l(\alpha) \leqq c_{0} l(\beta) \leqq c_{1}\left|z_{1}-z_{2}\right|,
$$

where $c_{1}=c_{0} \pi / 2$. Thus condition (1) is satisfied.

We note that the same reasoning shows that

$$
l(\alpha(z, w)) \leqq c_{0} l(\beta(z, w)) \text { if } z, w \in \alpha \cap \beta .
$$

Fix $z \in \alpha$. First, suppose $d(z, \partial D)=d(z, \partial U)$. Then $z \in \beta$ and using (7)

$$
\min _{j=1,2} l\left(\alpha_{j}\right) \leqq c_{1} d(z, \partial D),
$$

so in this case (2) is satisfied. Next, suppose $d(z, \partial D)=d\left(z, B_{k}\right)$ for some $k=1,2, \ldots$. We consider the possibilities $z \in \bar{A}_{k}, z \notin \bar{A}_{k}$ separately.

Suppose $z \in \bar{A}_{k}$. Then $k \in\{1,2\} \cup J$. If $k \in\{1,2\}$, then $\alpha_{k}=\sigma_{k}\left(z_{k}, z\right)$ and by Lemma 3.7

$$
l\left(\alpha_{k}\right) \leqq c_{2} d\left(z, B_{k}\right)=c_{2} d(z, \partial D),
$$

where $c_{2}=c_{2}(a, b)$. So (2) holds. On the other hand, suppose $k \in J-\{1,2\}$. Then $z \in \gamma_{k}$ and $d(z, \partial D)=d\left(C_{k}, B_{k}\right)=\varepsilon d_{k}$. Now for any $\zeta \in C_{k}$

whence by (7)

$$
d\left(\zeta, B_{0}\right) \leqq \varepsilon d_{k}+r_{k}+d_{k} \leqq(1+a+\varepsilon) d_{k},
$$

$$
\min _{j=1,2} l\left(\alpha\left(z_{j}, \zeta_{j k}\right)\right) \leqq c_{3} d(z, \partial D),
$$

where $c_{3}=c_{1}(1+a+\varepsilon) / \varepsilon=c_{3}(a, b)$. Since $l\left(\gamma_{k}\right) \leqq \pi r_{k} / 2 \leqq(\pi a / 2 \varepsilon) d(z, \partial D)$, (2) holds.

Finally, suppose $z \notin \bar{A}_{k}$. Then $z \in \beta$ and $d(z, \partial D)=d\left(z, B_{k}\right)>\varepsilon d_{k}$. If

then by (7)

$$
d\left(z, B_{0}\right) \leqq 2 h, \quad h=r_{k}+d_{k},
$$

while if

$$
\min _{j=1,2} l\left(\alpha_{j}\right) \leqq c_{0} \min _{j=1,2} l\left(\beta\left(z_{j}, z\right)\right) \leqq 2 c_{1} h \leqq 2 c_{1}(1+a) / \varepsilon d(z, \partial D),
$$

$$
n h \leqq d\left(z, B_{0}\right) \leqq(n+1) h \text { for some } n \geqq 2,
$$


then since $d(z, \partial D)=d\left(z, B_{k}\right) \geqq d\left(z, B_{0}\right)-h \geqq(n-1) h$, we conclude that

$$
\min _{j=1,2} l\left(\alpha_{j}\right) \leqq c_{1} d\left(z, B_{0}\right) \leqq c_{1}(n+1) h \leqq 3 c_{1} d(z, \partial D) .
$$

Thus in all cases (2) holds, so $D$ is uniform.

3.9. Corollary. Let $D^{\prime}=D-E$ where $D$ is uniform. Suppose there exists a positive constant $d$ such that

$$
\min _{j=1,2} d\left(\zeta_{j}, \partial D\right) \leqq d\left|\zeta_{1}-\zeta_{2}\right| \text { for all distinct points } \zeta_{1}, \zeta_{2} \in E
$$

Then $D^{\prime}$ is uniform with $c^{\prime}=c^{\prime}(c, d)$.

Proof. Fix $z_{1}, z_{2} \in D$. By [GO, Theorem 5] there exists a $K$-quasidisk $D_{0} \subset D$ with $z_{1}, z_{2} \in \bar{D}_{0}, K=K(c)$. Since $d\left(\zeta, \partial D_{0}\right) \leqq d(\zeta, \partial D)$ for $\zeta \in D_{0}$, it is easy to see that conditions (4) and (5) hold for the quasicircle domain $D_{0}^{\prime}=D_{0}-E$. Hence there exists an arc $\alpha$ in $D_{0}^{\prime} \subset D^{\prime}$ joining $z_{1}, z_{2}$ with $l(\alpha) \leqq c^{\prime}\left|z_{1}-z_{2}\right|$, and $\min _{j=1,2} l\left(\alpha_{j}\right) \leqq c^{\prime} d\left(z, \partial D_{0}^{\prime}\right) \leqq c^{\prime} d\left(z, \partial D^{\prime}\right)$ for all $z \in \alpha$, where $c^{\prime}=c^{\prime}(c, d)$.

\section{Hyperbolic geometry}

The euclidean conditions given in Theorems 1.1, 1.2 and in Corollary 3.9 can be stated in terms of the hyperbolic, or Poincaré, metric. The hyperbolic distance between two points $z_{1}, z_{2}$ in a domain $D \subset \overline{\mathbf{C}}$ with more than two boundary points is given by

$$
h_{D}\left(z_{1}, z_{2}\right)=h_{B}\left(\pi^{-1}\left(z_{1}\right), \pi^{-1}\left(z_{2}\right)\right)
$$

where $\pi: B \rightarrow D$ is a holomorphic projection, $B=\{w \in \mathbf{C}:|w|<1\}$ and $h_{B}\left(w_{1}, w_{2}\right)=$ $\log \left(\left|1-w_{1} \bar{w}_{2}\right|+\left|w_{1}-\bar{w}_{2}\right|\right) /\left(\left|1-w_{1} \bar{w}_{2}\right|-\left|w_{1}-\bar{w}_{2}\right|\right)$. If $D$ has at most two boundary points, then $h_{D} \equiv 0$. such that

4.1. Theorem. Suppose $D$ is uniform. Then there exists a constant $a=a(c)$

$$
h-\operatorname{dia}\left(B_{1}\right) \leqq a
$$

for any two components $B_{1}, B_{2}$ of $\overline{\mathbf{C}}-D$, where $h=h_{\overline{\mathbf{C}}-B_{2}}$.

Proof. Conditions (3) and (8) are equivalent.

4.2. Theorem. Let $B_{0}$ be any nondegenerate component of $\overline{\mathbf{C}}-D$. Suppose $D$ is a K-quasicircle domain and there exist constants $a, b$ such that

and

$$
h-\operatorname{dia}\left(B_{1}\right) \leqq a<+\infty
$$

$$
h\left(B_{1}, B_{2}\right) \geqq b>0
$$

for any two components $B_{1}, B_{2}$ of $\overline{\mathbf{C}}-D-B_{0}$, where $h=h_{\overline{\mathbf{C}}-B_{0}}$. Then $D$ is uniform with $c=c(a, b, K)$. 
Proof. Conditions (4), (5) and (9), (10) are equivalent.

4.3. Corollary. Let $D^{\prime}=D-E$ where $D$ is uniform. Suppose there exists a constant $d$ such that

$$
h_{D}\left(\zeta_{1}, \zeta_{2}\right) \geqq d>0 \quad \text { for all distinct points } \zeta_{1}, \zeta_{2} \in E .
$$

Then $D^{\prime}$ is uniform with $c^{\prime}=c^{\prime}(c, d)$.

\section{References}

[G1] Gehring, F. W.: Characteristic properties of quasidisks. - Séminaire de Mathématiques Supérieures 84. Les Presses de l’Université de Montréal, Montréal, 1982.

[G2] Gehring, F. W.: Injectivity of local quasi-isometries. - Comment Math. Helv. 57, 1982, $202-220$.

[G3] Gehring, F. W.: Uniform domains and the ubiquitous quasidisk. - Jahresbericht Deutsch. Math. Ver. 89, 1987, 88-103.

[GM] Gehring, F. W., and O. Martio: Quasiextremal distance domains and extension of quasiconformal mappings. - J. Analyse Math. 45, 1985, 181-206.

[GO] Gehring, F. W., and B. G. OsGood: Uniform domains and the quasihyperbolic metric. J. Analyse Math. 36, 1979, 50-74.

[G] GoluziN, G. M.: Geometric theory of functions of a complex variable. - Translations of Mathematical Monographs 26. American Mathematical Society, Providence, R. I., 1969.

[H] Hayman, W. K.: Multivalent functions. - Cambridge University Press, Cambridge, 1958.

[LV] Lehto, O., and K. I. VIRTANEN: Quasiconformal mappings in the plane. - Springer-Verlag, Berlin-Heidelberg-New York, 1973.

[MS] Martio, O., and J. Sarvas: Injectivity theorems in plane and space. - Ann. Acad. Sci. Fenn. Ser. A I Math. 4, 1978/1979, 383-401.

University of Cincinnati

Department of Mathematical Sciences

Cincinnati, Ohio 45221-0025

USA

Received 2 July 1986 